Severin Bühlmann

\section{Konzentrationsfaktoren - ein Buch mit sieben Siegeln}

Die Definition von Konzentrationsfaktoren ist an und für sich schon ein Thema, das längerer Betrachtungen bedürfte. Vorerst nur so viel:

(1) Der Konzentrationsfaktor wird in China ganz anders berechnet als in Taiwan. Das führt dazu, dass bereits von der Berechnungsart her grosse Unterschiede bestehen. Granulate aus China werden grob gesagt mit einem doppelt so hohen Konzentrationsfaktor angegeben als solche aus Taiwan. Würde ein einheitlicher Satz angewendet, würden also in etwa vergleichbare Zahlen herauskommen.

(2) Die in China angewandte Berechnungsmethode ist schlecht bzw. falsch. Die taiwanesische Methode stellt viel eher ein Abbild der Realität dar.

(3) Es konnte noch nirgends in Erfahrung gebracht werden, wie die Hersteller von Flüssigextrakten ihre Konzentrationsfaktoren berechnen. Weder Hersteller noch hiesige Distributoren weisen dazu irgendwelche Informationen aus.

(4) Aufgrund der folgenden Fakten ist davon auszugehen, dass die Anbieter von Flüssigextrakten ihre Botschaft als reine Marketingstrategie verbreiten und die Zahlen, wie im Verlauf dieses Beitrags bewiesen werden kann, vollkommen aus der Luft gegriffen sind, sofern man, was ja der Sinn ist, Konzentrationsfaktoren als ein

\title{
Rohdrogen, Granulate oder flüssige Extrakte: Was wirkt am besten? - Teil 2
}

Mass nimmt, wie viel mehr Inhaltsstoffe in einem Extrakt gegenüber dem Standarddekokt vorhanden sind.

Das Brion Research Institute in Taiwan bekam von uns den Auftrag, Granulate und Flüssigextrakte auf gewisse Parameter zu untersuchen. Leitsubstanzen sind Inhaltsstoffe von Pflanzen, die als für die klinische Wirkung besonders wichtig erachtet werden und/oder in grösseren Mengen vorhanden sind. Selbstverständlich erwartet man bei einer Konzentrierung einen entsprechend hohen Wert an solchen Substanzen.

Weiter wollten wir wissen, was denn sonst noch in Flüssigextrakten enthalten ist und welche Bedeutung oder Auswirkungen Zusatzstoffe haben.

Den Untersuchungsauftrag bekam das Labor des Brion Research Institute, weil es in Europa kein Labor gibt, das diese schwierigen Analysen routinemässig durchführen könnte. Auch Universitäten in Europa verfügen nicht a priori über die hierfür notwendigen Methoden, sondern müssen diese in langwierigen Evaluationsverfahren erarbeiten. Für einen einzelnen Inhaltsstoff kann dies bereits Monate in Anspruch nehmen. Das Brion Research Institute entwickelte über Jahrzehnte diese Methoden und kann innert nützlicher Frist Resultate liefern.

Die Resultate bezüglich der Leitsubstanzen sind in Tabelle 1 zusammengefasst. Die letzte Spalte zeigt, wie viel Mal schwächer die Konzentration von wichtigen Inhaltsstoffen im Flüs- sigextrakt im Vergleich zum Granulat ist.

\section{Quantitative \\ Inhaltsstoffanalysen an Flüssigextrakten im Vergleich zu Granulaten}

Im Fall von Bai Shao (Paeonia lactiflora) findet man einen eklatanten Unterschied zwischen den in der Schweiz sich im Handel befindlichen Flüssigextrakten und SunTen-Granulaten (ST), die von Complemedis vertrieben werden (Tab. 2): Der Gehalt des klinisch wichtigen Inhaltsstoffes Paeoniflorin liegt bei Complemedis bei $19,53 \mathrm{mg} / \mathrm{g}$ - entsprechend einem Gehalt von $1,95 \%$ - und beim Flüssigextrakt bei $0,41 \mathrm{mg} / \mathrm{g}$ - entsprechend $0,04 \%$. Complemedis weist also fast $50-\mathrm{mal}$ mehr von diesem notwendigen Stoff auf, wogegen dieser beim Flüssigextrakt sozusagen nicht vorhanden ist. Die chinesische Pharmakopöe schreibt jedoch einen Mindestgehalt von nicht weniger als 1,2\% Paeoniflorin vor.

Bei Süssholz (= Gan Cao (Glycyrrhi$z a)$ ) zeigt das Flüssigprodukt eine mehr als 20-fach schlechtere Konzentration an Liquiritin und einen 8 -fach schlechteren Wert für Glyzyrrhizin. Die chinesische Pharmakopöe fordert nicht weniger als $0,5 \%$ Liquiritin und 2\% Glyzyrrhizin. Das Flüssigextrakt erreicht diese Werte mit $0,12 \%$ Liquiritin und $0,51 \%$ Glyzyrrhizin bei Weitem nicht, während das Granulat die geforderte Minimalmenge deutlich übertrifft.

\section{KARGER}

(c) 2016 S. Karger GmbH, Freiburg 
Tab. 1. Quantitative Inhaltsstoffanalysen an Flüssigextrakten im Vergleich zu Granulaten

\begin{tabular}{|c|c|c|c|c|c|}
\hline \multirow[t]{2}{*}{ Pinyin } & \multirow[t]{2}{*}{ Pharmazeutisch } & \multirow[t]{2}{*}{ Inhaltsstoff } & \multicolumn{2}{|l|}{ Resultat } & \multirow{2}{*}{$\begin{array}{l}\text { Differenz, } \\
\text { gerundet }\end{array}$} \\
\hline & & & Granulat & Flüssigextrakt & \\
\hline Tian Ma & Gastrodiae Rhizoma & Gastrodin & 2,92 & 1,63 & $2 \times$ \\
\hline Bai Zhu & Atractylodes macrocephalae Rhizoma & Atractylenolid III & 0,44 & 0,05 & $9 \times$ \\
\hline \multirow[t]{2}{*}{ Sheng Jiang } & \multirow[t]{2}{*}{ Zingiberis Rhizoma recens } & 6-Gingerol & 2,64 & 0,04 & $53 \times$ \\
\hline & & 6-Shogaol & 1,15 & 0,02 & $57 \times$ \\
\hline \multirow[t]{2}{*}{ Sheng Di Huang } & \multirow[t]{2}{*}{ Rehmanniae glutinosae Radix et Rhizoma } & Catalpol & 1,97 & 0,30 & $7 \times$ \\
\hline & & Stachyose & 54,49 & 18,31 & $3 \times$ \\
\hline \multirow[t]{2}{*}{ Ban Xia } & \multirow[t]{2}{*}{ Pinelliae Rhizoma praeparata } & Adenin & 0,059 & 0,002 & $30 \times$ \\
\hline & & Guanosin & 0,016 & 0,002 & $8 \times$ \\
\hline \multirow[t]{2}{*}{ Gan Cao } & \multirow[t]{2}{*}{ Glyzyrrhizae Radix } & Liquiritin & 27,17 & 1,17 & $23 \times$ \\
\hline & & Glyzyrrhizin & 42,88 & 5,05 & $8 \times$ \\
\hline \multirow[t]{5}{*}{ Zhi Gan Cao } & \multirow[t]{5}{*}{ Glyzyrrhizae Radix melle tosta } & Liquiritin & 29,31 & 1,91 & $15 \times$ \\
\hline & & Glyzyrrhizin & 39,41 & 2,15 & $7 \times$ \\
\hline & & Fructose & 47,45 & 5,81 & $8 \times$ \\
\hline & & Glucose & 30,43 & 7,59 & $4 \times$ \\
\hline & & Saccharose & 89,70 & 15,11 & $6 \times$ \\
\hline \multirow[t]{2}{*}{ Mu Dan Pi } & \multirow[t]{2}{*}{ Moutan Cortex } & Paeoniflorin & 11,53 & 0,22 & $52 \times$ \\
\hline & & Paeonol & 8,67 & 0,08 & $108 \times$ \\
\hline Bai Shao & Paeoniae Radix & Paeoniflorin & 19,53 & 0,41 & $48 \times$ \\
\hline \multirow{2}{*}{ Du Zhong } & \multirow{2}{*}{ Eucommiae Cortex } & Chlorogensäure & 0,69 & 0,02 & $35 \times$ \\
\hline & & Pinoresinaldiglucosid & 0,75 & 0,09 & $8 \times$ \\
\hline \multirow[t]{6}{*}{$\mathrm{Fu} \mathrm{Zi}$} & Aconiti Radix praeparata & Aconitin & 0 & 0 & \\
\hline & & Hypaconitin & 43,67 & 4,58 & $10 \times$ \\
\hline & & Mesaconitin & 23,81 & 0 & $!$ \\
\hline & & Benzoylaconin & 25,55 & 0,71 & $36 \times$ \\
\hline & & Benzoylhypaconin & 19,55 & 7,21 & $3 \times$ \\
\hline & & Benzoylmesaconin & 240,22 & 28,00 & $9 \times$ \\
\hline Chai Hu & Bupleuri Radix & Saikosaponin A & 2,12 & 0,13 & $16 \times$ \\
\hline & & Saikosaponin C & 0,41 & 0,13 & $3 \times$ \\
\hline & & Saikosaponin D & 0,05 & 0,02 & $2,5 \times$ \\
\hline Sha Ren & Crataegi Fructus & Quercetrin & 0,056 & 0,004 & $14 \times$ \\
\hline Gui Zhi & Cinnamomi Ramuli & Zimtsäure & 0,81 & 0,02 & $40 \times$ \\
\hline & & Cinnamaldehyd & 2,05 & 0,03 & $68 \times$ \\
\hline & & 2-Methoxy-Cinnamaldehyd & 0,73 & 0,01 & $73 \times$ \\
\hline Huang Qi & Astragali Radix & Calyconsin-7-O-Glucosid & 0,26 & 0,12 & $2 \times$ \\
\hline & & Astragalosid IV & 0,12 & 0,02 & $6 x$ \\
\hline & & Astragalosid IV (hydrolysiert) & 1,58 & 0,06 & $26 \times$ \\
\hline Huang Qin & Scutellariae Radix & Baicalin & 133,62 & 0 & $!$ \\
\hline & & Baicalein & 7,07 & 0,14 & $50 \times$ \\
\hline Dang Gui & Angelicae sinensis Radix & Ferulasäure & 0,68 & 0,08 & $8,5 \times$ \\
\hline & & Ligustilid & 2,66 & 0,14 & $19 \times$ \\
\hline Suan Zao Ren & Ziziphi Semen & Spinosin & 0,17 & 0,13 & $1,3 \times$ \\
\hline & & Jujubosid A & 0,11 & 0,03 & $4 \times$ \\
\hline & & Jujubosid B & 0,06 & 0,02 & $3 \times$ \\
\hline Shu Di Huang & Rehmanniae praeparatae Radix et Rhizoma & Catalpol & 0 & 0 & \\
\hline & & Stachyose & 13,69 & 4,22 & $3 \times$ \\
\hline & & Process Level Index & 6,21 & 5,73 & $=$ \\
\hline Dang Shen & Salviae Radix & Lobetyolin & 0,39 & 0,02 & $20 \times$ \\
\hline Ren Shen & Ginseng Radix & Ginsenosid Rg1 & 1,32 & 0,87 & $1,5 \times$ \\
\hline & & Ginsenosid Re & 1,01 & 1,1 & $=$ \\
\hline & & Ginsenosid Rb1 & 4,26 & 2,69 & $1,5 \times$ \\
\hline
\end{tabular}

\section{Weitere Bestandteile von Flüssigextrakten: Glycerin und Alkohol}

Der Hersteller von Flüssigextrakten und sein lokaler Distributor berichten, dass diese im ersten Schritt des Extraktionsprozesses mittels alkoholischer Mazeration gewonnen werden; danach wird extrahiert und am Schluss werden Konservierungsstoffe beigegeben (Tab. 3). Der stark süsse Geschmack kommt vom Konservierungsstoff Glycerin. Wir liessen auf Glycerin und Alkohol testen und fanden Anteile von 45-62\% Glycerin, aber auch Alkohol von 0 (N.D. = not detectable) bis $3 \%$. 
Tab. 2. Bestimmung von chemischen Leitsubstanzen in Flüssig extrakten und Granulaten mittels HPLC/ PDA

\begin{tabular}{|c|c|c|c|c|c|c|c|}
\hline \multicolumn{2}{|c|}{ Name der Probe } & \multirow[t]{2}{*}{ Probennummer } & \multirow[t]{2}{*}{ Nachweis (HPLC Fingerprint) } & & \multicolumn{3}{|l|}{ Leitsubstanz } \\
\hline & & & & & Stoff & $\mathrm{mg} / \mathrm{g}$ & $\%$ \\
\hline \multirow[t]{5}{*}{ Bai Shao } & 白芶 & 80495934-3 & Albiflorin & + & Paeoniflorin & 0,41 & 0,04 \\
\hline & & & Paeoniflorin & + & & & \\
\hline & & & Paeoniflorinsulfonat & + & & & \\
\hline & 白苟 & Lot\#13050605 & Albiflorin & + & Paeoniflorin & 19,53 & 1,95 \\
\hline & $(\mathrm{ST})$ & & Paeoniflorin & + & & & \\
\hline \multirow[t]{4}{*}{ Gan Cao } & 甘草 & 80495934-7 & Liquiritin & + & Liquiritin & 1,17 & 0,12 \\
\hline & & & Glycyrrhizin & + & Glycyrrhizin & 5,05 & 0,51 \\
\hline & 甘草 & Lot\#13041004 & Liquiritin & + & Liquiritin & 25,46 & 2,55 \\
\hline & $(\mathrm{ST})$ & & Glycyrrhizin & + & Glycyrrhizin & 32,86 & 3,29 \\
\hline
\end{tabular}

Tab. 3. Bestimmung von Ethanol und Glycerin in Pflanzenextrakten mittels GC/ MS und HPLC/RI

\begin{tabular}{lllll}
\hline \multirow{2}{*}{ Name der Probe } & Probennummer & \multicolumn{2}{l}{ GC/MS } & HPLC/RI \\
\cline { 4 - 5 } & & & Ethanol, \% & Glycerin, \% \\
\hline Bai Shao & 白䓎 & $80495934-3$ & 3,02 & 46,2 \\
Gan Cao & 甘草 & $80495934-7$ & 0,55 & 46,0 \\
Huang Qin & 黃芩 & $80495947-14$ & N.D. & 45,2 \\
\multirow{2}{*}{ Ren Shen } & 人參 & $80495947-2$ & LOD $=0,1$ & \\
& & & N.D. & 48,3 \\
\multirow{2}{*}{ Dang Gui } & 當歸 & $80495934-4$ & 0,87 & 54,0 \\
\hline
\end{tabular}

LOD = Limit of detection (Nachweisgrenze); N.D. = not detectable.
Glycerin ist ein Stoff, den wohl die meisten zuerst mit Autofahren im Winter in Verbindung bringen, weil man ihn als Frostschutzmittel einsetzt. Inwieweit Glycerin in der Traditionellen Chinesischen Medizin (TCM) etwas zu suchen hat, sei dahingestellt. Die Schulmedizin macht jedenfalls darauf aufmerksam, dass Glycerin eine Verstoffwechselung durchmacht. Lassen wir für einmal die englische Internetseite von Wikipedia sprechen:

«Für die menschliche Ernährung wird Glycerin durch die FDA der USA unter den Alkoholen als kalorischer Nährstoff klassifiziert. Die American Dietetic Association zählt Glycerin zu den Kohlehydraten.»

\section{Zucker in Flüssigextrakten}

Die nächste Frage war, ob allenfalls weitere Zucker als Konservierungsmittel in Flüssigextrakten eingesetzt werden. Hier das Resultat: Zellulose war der einzige zusätzliche Zucker, den wir in ausgewählten Produkten fanden. Auffallend ist der hohe Anteil in Ren Shen (Ginseng) und in Dang Gui (Angelica sinensis) (Tab. 4).

\section{Parabene als \\ Konservierungsstoffe}

Etwas sorgenvoller dürften TCMExperten bzw. ihre Patienten die folgenden Resultate betrachten: Parabene werden in den geprüften Flüssigextrakten reichlich gefunden (Abb. 1)

Parabene sind Konservierungsstoffe, welche in Nahrungsmitteln nicht gern gesehen werden. Sie wirken kanzerogen, und seriöse Hersteller weichen auf andere Konservierungsstoffe aus. Konsumentenorganisationen warnen vor diesen Stoffen. Zwei dieser Parabene (PHB und PK1) werden in Tabelle 5 als Summe dargestellt.

\section{Weitere unerfreuliche Inhaltsstoffe}

Ein interessantes Bild zeigt sich bezüglich der HPLC Fingerprints von Flüssigextrakten und Granulaten
(Abb. 2). Hier sehen wir, dass für die Produktion der Flüssigextrakte geschwefelte Rohdrogen verwendet wurden. Die Schwefelung macht die Rohdrogen haltbarer, aber auch dieses Verfahren wird von den Konsumenten abgelehnt. Geschwefelte Ware kann bei empfindlichen Menschen Kopfschmerzen verursachen. Ausserdem verhält sich der Schwefel nicht inert, sondern geht mit den Inhaltsstoffen der Kräuter Verbindungen ein! So wird er mit Paeoniflorin, einem wichtigen Inhaltsstoff von Bai Shao $(P$. lactiflora), zu Paeoniflorinsulfonat, was natürlich nicht erwünscht ist, denn so geht eine wichtige Wirkkomponente verloren. Nach diesem Resultat haben wir als Auftraggeber nicht gesucht, sondern das Brion Research Institute hat uns darauf aufmerksam gemacht. An diesem Beispiel zeigt sich die jahrzehntelange Erfahrung der taiwanesischen Hersteller, die gegenüber anderen Produzenten ein Know-how angesammelt haben, das die Qualität von Granulaten aus Taiwan einmal mehr beweist. 
Tab. 4. Bestimmung von Zuckern in Flüssigextrakten mittels HPLC/ELSD

\begin{tabular}{|c|c|c|c|c|c|c|c|c|}
\hline \multicolumn{2}{|c|}{ Name der Probe } & \multirow[t]{2}{*}{ Probennummer } & \multicolumn{4}{|c|}{ Zuckerprofil, mg/g } & \multicolumn{2}{|c|}{ Total Zucker } \\
\hline & & & Saccharose & Fruktose & Glucose & Cellobiose & $\mathrm{mg} / \mathrm{g}$ & $\%$ \\
\hline Bai Shao & 白苻 & 80495934-3 & N.D. & N.D. & N.D. & 5,92 & 5,92 & 0,59 \\
\hline Gan Cao & 甘草 & 80495934-7 & N.D. & N.D. & N.D. & 5,92 & 5,92 & 0,59 \\
\hline Huang Qin & 黃芩 & 80495947-14 & N.D. & N.D. & N.D. & 9,46 & 9,46 & 0,95 \\
\hline Ren Shen & 人參 & $80495947-2$ & N.D. & N.D. & 6,29 & 37,08 & 43,37 & 4,34 \\
\hline Dang Gui & 當歸 & 80495934-4 & N.D. & N.D. & N.D. & 36,33 & 36,33 & 3,63 \\
\hline
\end{tabular}

N.D. $=$ Not detectable.
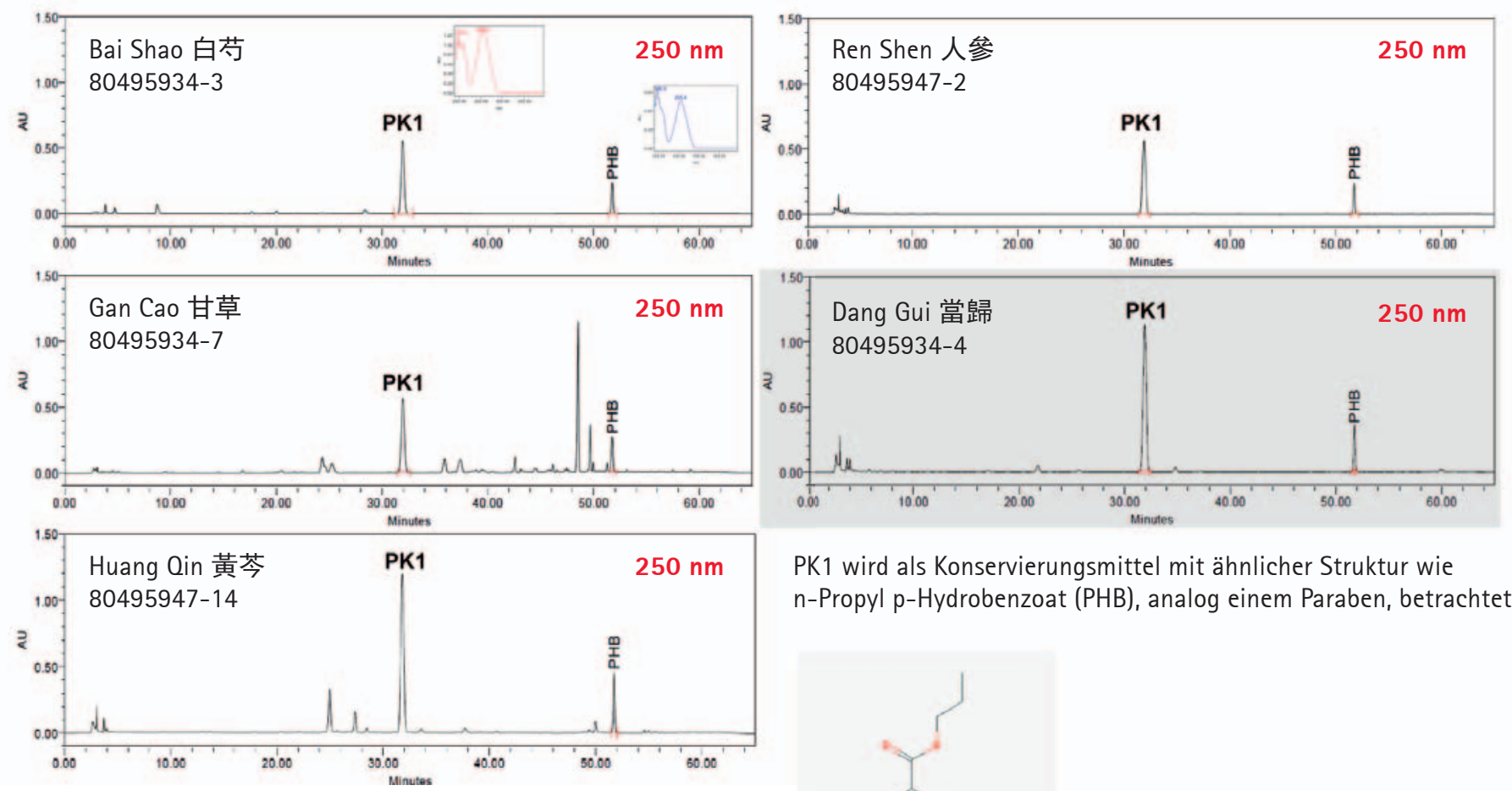

PK1 wird als Konservierungsmittel mit ähnlicher Struktur wie n-Propyl p-Hydrobenzoat (PHB), analog einem Paraben, betrachtet.

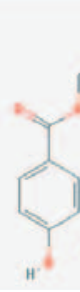

PHB

Abb. 1. HPLC Fingerprints zur Bestimmung von Konservierungsstoffen in Flüssigextrakten.

\section{Weitere Argumente für oder gegen Flüssigextrakte}

Nachdem bereits der Aspekt «Konzentrationsfaktor» ausführlich diskutiert wurde, können teilweise auch die weiteren Anpreisungen der Anbieter von Flüssigextrakten in Anlehnung an die gesammelten Daten diskutiert werden.

\section{Wirksamkeit}

Es ist offensichtlich, dass ein Vergleich der Wirksamkeit von Granula- ten oder Flüssigextrakten davon abhängt, ob die Qualität der Mittel gut ist und auch genügend Wirkstoffe in diesen enthalten sind, sodass ein Effekt erzielt wird, der über dem einer Placebowirkung steht. Letztere erklärt wahrscheinlich, warum gewisse Flüssigextrakte trotzdem wirken, denn die Placebowirkung ist oft beträchtlich gut. Natürlich besteht eine Dosis-Wirkungs-Beziehung - und diese kann bei Kenntnis der Konzentrationsfaktoren und weiterer qualitativ entscheidender Parameter berechnet werden. Einschränkend muss gesagt werden, dass man die Dosis ins Unermessliche steigern kann und trotzdem keine klinische Wirkung erreicht werden kann, wenn ein Produkt schlicht praktisch nichts enthält ausser Konservierungsund Füllmitteln.

\section{Compliance}

Die Geschmäcker sind verschieden. Es liegt auf der Hand, dass Flüssigprodukte unter Umständen eine gute Akzeptanz haben können. Im ersten Moment kommen einem viele Vorteile in den Sinn. Bei näherem Hinsehen ver- 
Flüssigextrakte
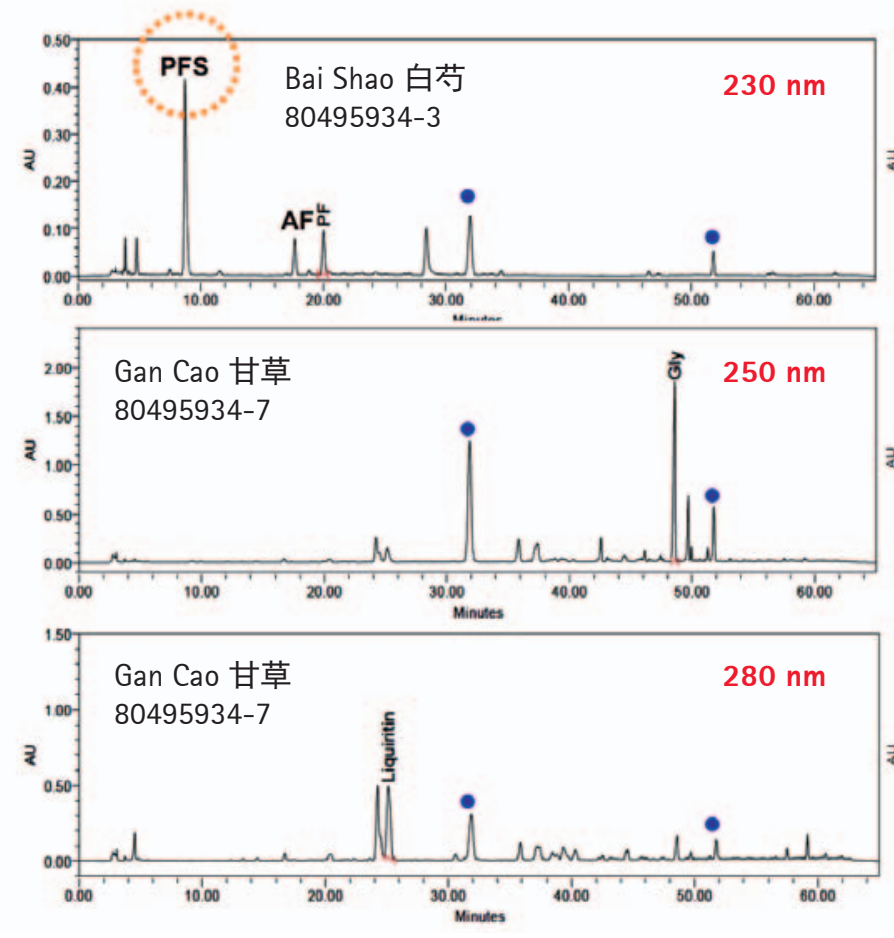

\section{Granulate}
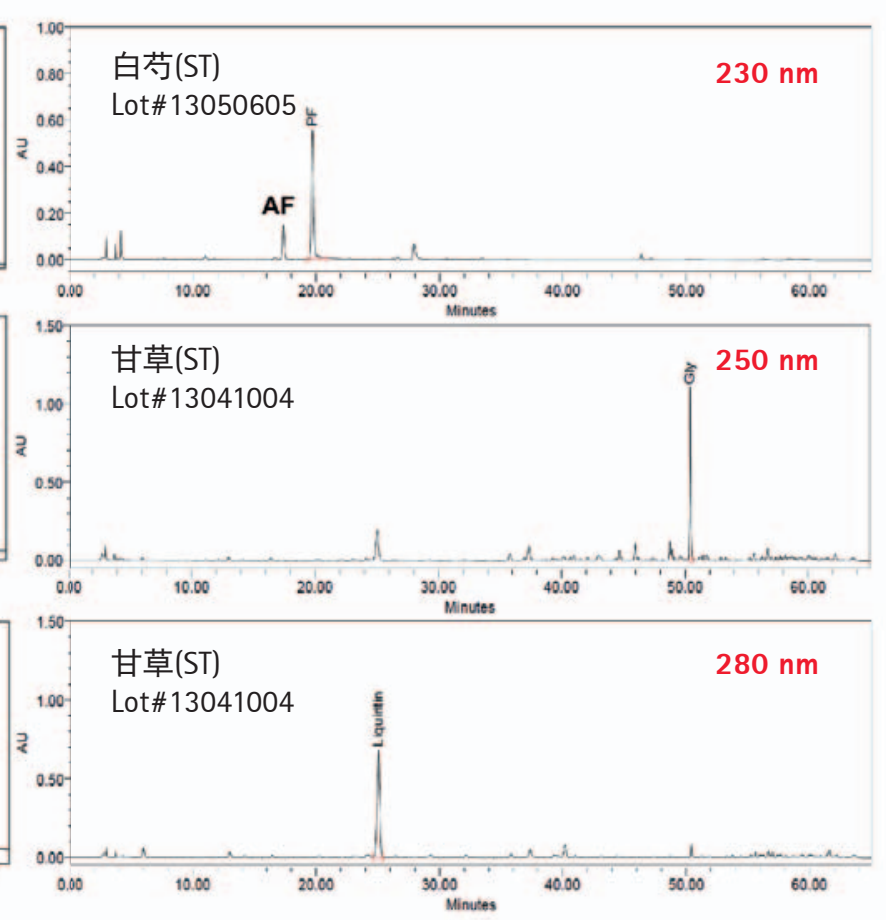

- Peak der Konservierungsstoffe

Abb. 2. HPLC Fingerprints von Flüssigextrakten und Granulaten. AF = Albiflorin; PF = Paeoniflorin; PFS = Paeoniflorinsulfonat (PFS) (entsteht aus Paeoniflorin nach Schwefelung); Gly = Glyzyrrhizin.

flüchtigen diese sich aber schnell, und es bleibt bei suggestiven Unterstellungen. Die Süsse von Glycerin, mit dem Flüssigextrakte hierzulande stabilisiert werden, wird von einigen Patienten, vielleicht vor allem Kindern, gemocht. Anderen ist sie vollkommen zuwider. Den chemischen Nachgeschmack empfinden viele als schwer ertragbar. Will man ihn abschwächen, muss man mit viel Wasser verdünnen. Zudem stellt sich die Frage, ob diese alles übertönende Geschmacksnote nicht doch den klinisch erwünschten Erfolg trübt. Immer wieder kann zudem festgestellt werden, dass Patienten, denen aufgrund der TCM-Diagnose eine überaus bittere Medizin gegeben werden muss, diese sogar als geschmacklich hervorragend empfinden und geradezu danach lechzen, wahrscheinlich weil ihre Situation genau das erfordert. Bezeichnet ein Patient seine Mixtur als eklig oder ist er aus geschmacklichen Gründen nicht

Tab. 5. Bestimmung von Konservierungsstoffen in Flüssigextrakten mittels HPLC/PDA

\begin{tabular}{llllll}
\hline Name der Probe & Probennummer & \multicolumn{2}{l}{ Konservierungsstoffe, mg/g } \\
\cline { 4 - 6 } & & & PHB & PK1 ${ }^{\mathrm{a}}$ & $\begin{array}{l}\text { total (PHB + } \\
\text { PK1) }\end{array}$ \\
\cline { 4 - 6 } & & & & 1,09 & 1,32 \\
Bai Shao & 白药 & $80495934-3$ & 0,23 & 1,13 & 1,41 \\
Gan Cao & 甘草 & $80495934-7$ & 0,28 & 1,1 & 1,31 \\
Huang Qin & 黃芩 & $80495947-14$ & 0,21 & 1,1 & 1,33 \\
Ren Shen & 人參 & $80495947-2$ & 0,23 & 1,11 & 1,28 \\
Dang Gui & 當歸 & $80495934-4$ & 0,17 & 1,11 & \\
\hline
\end{tabular}

${ }^{a}$ Berechnet auf Basis der PHB-Eichkurve.

in der Lage, diese einzunehmen, muss die Diagnose überdacht werden. $\mathrm{Zu}$ dem sei noch darauf hingewiesen, dass Flüssigextrakte im Handgepäck bei Flugreisen ein Problem sein können.

\section{Preisgestaltung}

Manche Patienten schätzen die Tatsache, dass Flüssigextrakte immer den gleichen Preis haben. Aufgrund der obigen Messungen kann gesagt werden, dass damit für den Hersteller auch kein grosses Risiko verbunden ist. Wenn Granulate je nach Produkt unterschiedliche Preise haben, so zeigt das transparent, welche Kräuter wertvoll und teuer sind. Es ist zu hoffen, dass dies zu einer höheren Wertschätzung bei den Verschreibenden und den Patienten sowie zu einem sorgsamen Umgang mit den vorhandenen Ressourcen führt. In Anbetracht aller Faktoren können Granulate als die finanziell günstigste Lösung ausgewiesen werden. 